
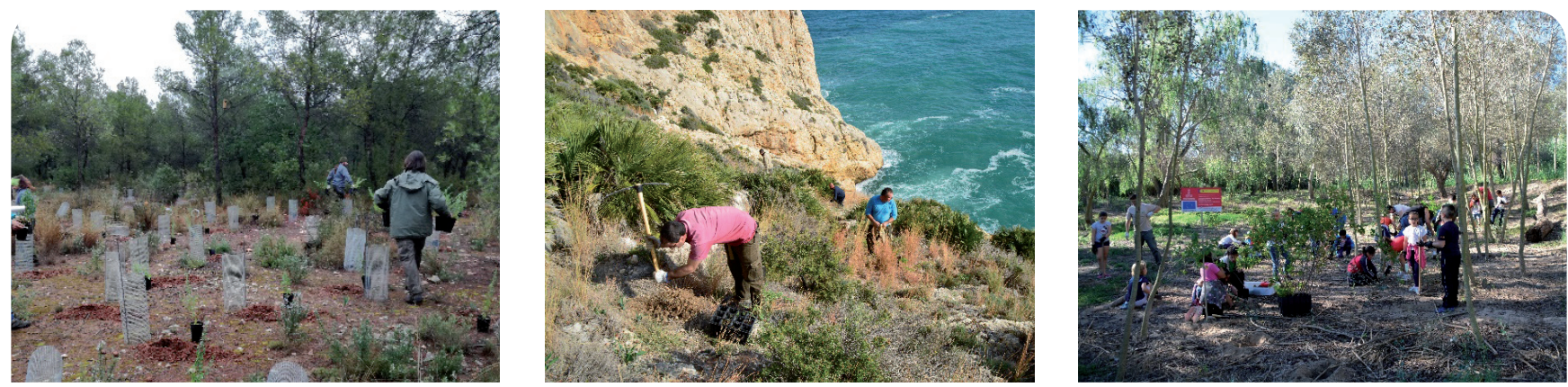

Figura 2. Trabajos de plantación realizados en el marco del Plan de recuperación de Cistus heterophyllus en el LIC Serra Calderona (izquierda)، de Medicago citrina en el LIC Penyasegats de la Marina (centro), y de Frangula alnus en el Paraje Natural La Chopera de Algemesí (derecha).



口EPE

$\square \mathrm{VU}$

$\square$ PNC

$\square \mathrm{VI}$

m Flora estructura

Figura 3. Producción de planta durante el periodo julio 2017-junio 2018 según la categoría de protección de las especies de flora (Orden 1/2013) EPE: En peligro de extinción; VU: Vulnerable; PNC: Protegida no catalogada; VI: Vigilada.

\section{Agradecimientos}

A los compañeros del CIEF y de las asistencias técnicas de Servicios Territoriales y Brigadas Natura 2000 de las provincias de Valencia, Castellón y Alicante, por su colaboración y trabajo.

INMACULADA FERRANDO-PARDO ${ }^{1,2}$, P. PABLO FERRER-GALLEGO ${ }^{1,2}$ VICENTE DEL TORO ${ }^{2,3}$, FRANCISCO J. ALBERT ${ }^{1,2}$ EMILIO LAGUNA ${ }^{1,3}$ \& JUAN JIMÉNEZ ${ }^{3}$

1. Generalitat Valenciana. Servicio de Vida Silvestre - Centro para la Investigación y Experimentación Forestal (CIEF). Avda. Comarques del País Valencià, 114. 46930 Quart de Poblet, Valencia.

E-mail: endemica_cief@gva.es 2. Generalitat Valenciana. VAERSA.

Avda de les Corts Valencianes, 20,46015 Valencia. 3. Generalitat Valenciana. Servicio de Vida Silvestre. c/ Castán Tobeñas, 77. Ciutat administrativa 9 de octubre-Torre 1. 46018 Valencia.

\title{
Seguimiento de las poblaciones de dos especies perennes de interés Do: 10.1536// 2018.22 .005 para la conservación en el Delta del Llobregat
}

El seguimiento periódico de poblaciones es una medida habitual en la conservación in situ de especies amenazadas, si bien su uso no está generalizado en el caso de las plantas y especialmente en el de las perennes, cuya demografía exige seguimientos a largo plazo. El presente trabajo recoge los datos de dos seguimientos consecutivos -separados por más de una década- realizados sobre dos especies amenazadas en el Delta del Llobregat (Barcelona): Kosteletzkya pentacarpa (L.) Ledeb. y Orobanche foetida Poir.

Kosteletzkya pentacarpa (Malvaceae) en España crece principalmente en los deltas del Llobregat y del Ebro y la Albufera de Valencia. En el Delta del Llobregat habita únicamente en las marismas de la laguna de la Ricarda (Pino \& de Roa, 2003). Está catalogada como vulnerable en Europa y Cataluña (Sáez et al., 2010; Buord et al., 2011) e incluida en la Directiva Hábitats (92/43/CEE), el Convenio de Berna (1979), el Catálogo Español de Especies Amenazadas (Real Decreto 139/2011) y el Catálogo de flora amenazada de Cataluña (Decreto 172/2008). Orobanche foetida habita en las dunas litorales fijadas, donde parasita exclusivamente a Ononis natrix. Se distribuye por gran parte de la costa mediterránea de la península lbérica, si bien se considera en peligro de extinción en Cataluña (Decreto 172/2008). Antiguamente se extendía por la costa sur catalana, pero los datos recientes se limitan al Delta del Llobregat, donde es bastante rara (González et al., 2016).

Las poblaciones del Delta del Llobregat de ambas especies fueron prospectadas a finales del siglo XX e inicios del XXI. K. pentacarpa lo fue entre 1998 y 1999 (Pino \& de Roa, 2003) en las marismas de la laguna de la Ricarda, donde ya se conocía su existencia. También se visitaron otras lagunas con ambientes similares (el Remolar, Ca l'Arana, etc.) con resultados negativos. Las poblaciones de $O$. foetida fueron prospectadas en 2003 (Pino, 2003) a lo largo de los 18 km de playas del hemidelta sur del Llobregat. En primavera-verano de 2018, después de 20 y 15 años respectivamente, se repitieron dichos muestreos.

En el caso de $K$. pentacarpa la prospección de 1998-1999 arrojó un total de 684 individuos repartidos en una decena de núcleos. El censo de 2018 fue de 743 individuos, lo que representa un leve incremento de aproximadamente el $8 \%$, un resultado en la línea del crecimiento sostenido que se observó en estudios de la dinámica poblacional de algunos de los núcleos entre 1995 y 2004 (Pino \& de Roa, 2007). Los principales núcleos poblacionales han permanecido estables 
Figura 1:

Flor y fruto de

Kosteletzkya pentacarpa

en la Laguna de la

Ricarda, septiembre de

2018 (Foto L. Fuentes).

Tallos de Orobanche foetida sobre su huésped Ononis natrix, Espacio protegido de Cal Francès, mayo de 2018 (Foto: L. Fuentes)

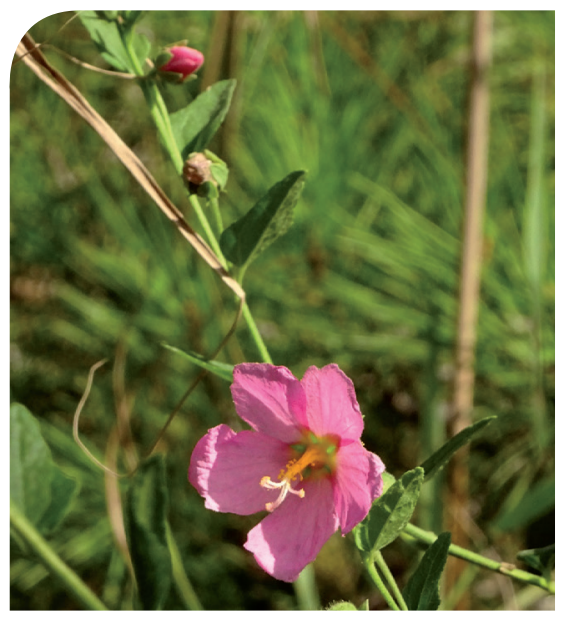

durante los últimos 20 años: sólo algunos núcleos de reducido tamaño han desaparecido o están a punto de hacerlo (Figura 2). También se han encontrado algunos núcleos nuevos o que no fueron detectados en 1998-1999.

En cuanto a O. foetida, el censo de 2003 arrojó un total de 380 individuos, repartidos en 9 poblaciones en el Delta del Llobregat (Pino, 2003). En los muestreos de 2018 se ha confirmado la persistencia de sólo 5 de ellas (Figura 3), sumando un total de 359 individuos. Fuera de los espacios protegidos, las poblaciones se han visto reducidas o han desaparecido, dos de ellas debido a la ampliación del Aeropuerto del Prat, mientras que las cuatro poblaciones situadas en espacios protegidos han mostrado tendencias diferentes: la población principal cuenta con 288 individuos, más del doble que en 2003, otra población se ha reducido sustancialmente (de 20 individuos en 2003 a sólo 6 en la actualidad) y las otras dos no han sido reencontradas en 2018.

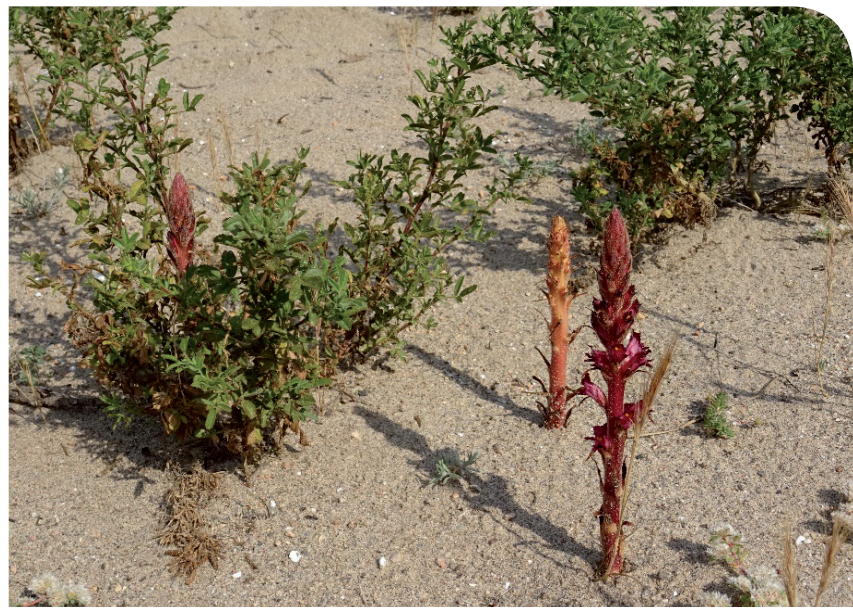

Los resultados permiten constatar la notable resiliencia de las especies estudiadas, que han mantenido gran parte de sus núcleos poblacionales iniciales e incluso el total de individuos. En el caso de K. pentacarpa, probablemente se deba a la elevada longevidad y supervivencia de los individuos adultos, como se demostró en un estudio poblacional (Pino et al., 2007). También se hace evidente la necesidad de conservar los hábitats de interés comunitario en los que se encuentran estas especies, manteniendo un ambiente suficientemente estable e inalterado, tal como respalda la mayor persistencia de los núcleos situados en espacios protegidos, en particular los de $K$. pentacarpa, respecto a la de los núcleos de $O$. foetida repartidos por el litoral del Delta, bajo condiciones de conservación muy distintas. La continuación de estudios a largo plazo será necesaria para conocer la evolución de las poblaciones e identificar otros posibles factores de amenaza.

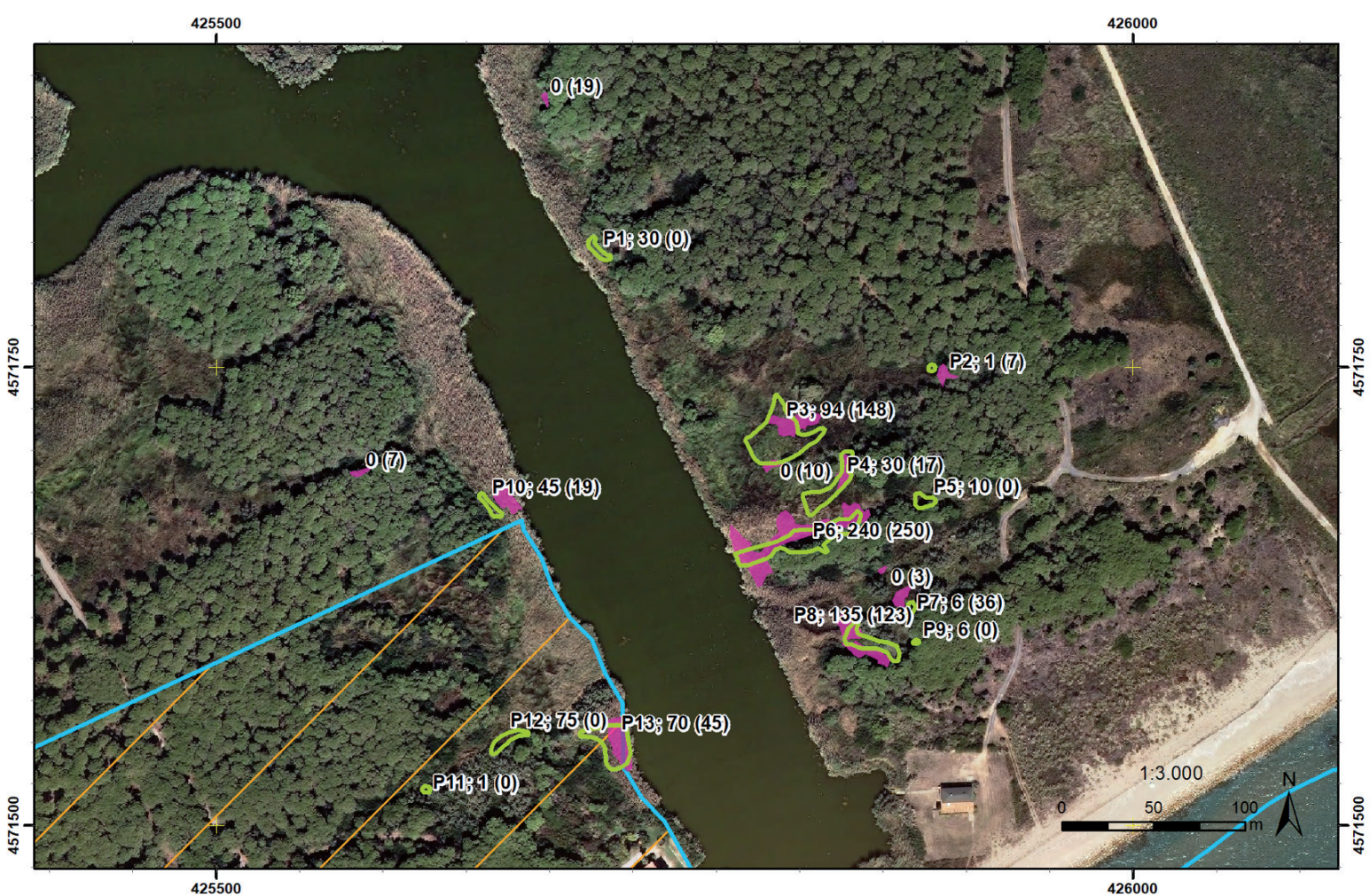

Figura 2. Mapa de la población de Kosteletzkya pentacarpa en la Laguna de la Ricarda. Los polígonos indican los límites aproximados de los núcleos poblacionales (P1-P13) detectados en las prospecciones de 2018 (verde) y en las prospecciones de 1998-1999 (fucsia). Los números indican el censo de individuos aproximado de cada núcleo poblacional en 2018 y, entre paréntesis, el censo en 1998-1999. La línea azul separa el límite entre la Reserva natural parcial (toda la superficie visible) y la Zona periférica de la reserva natural parcial (zona con trama naranja). 


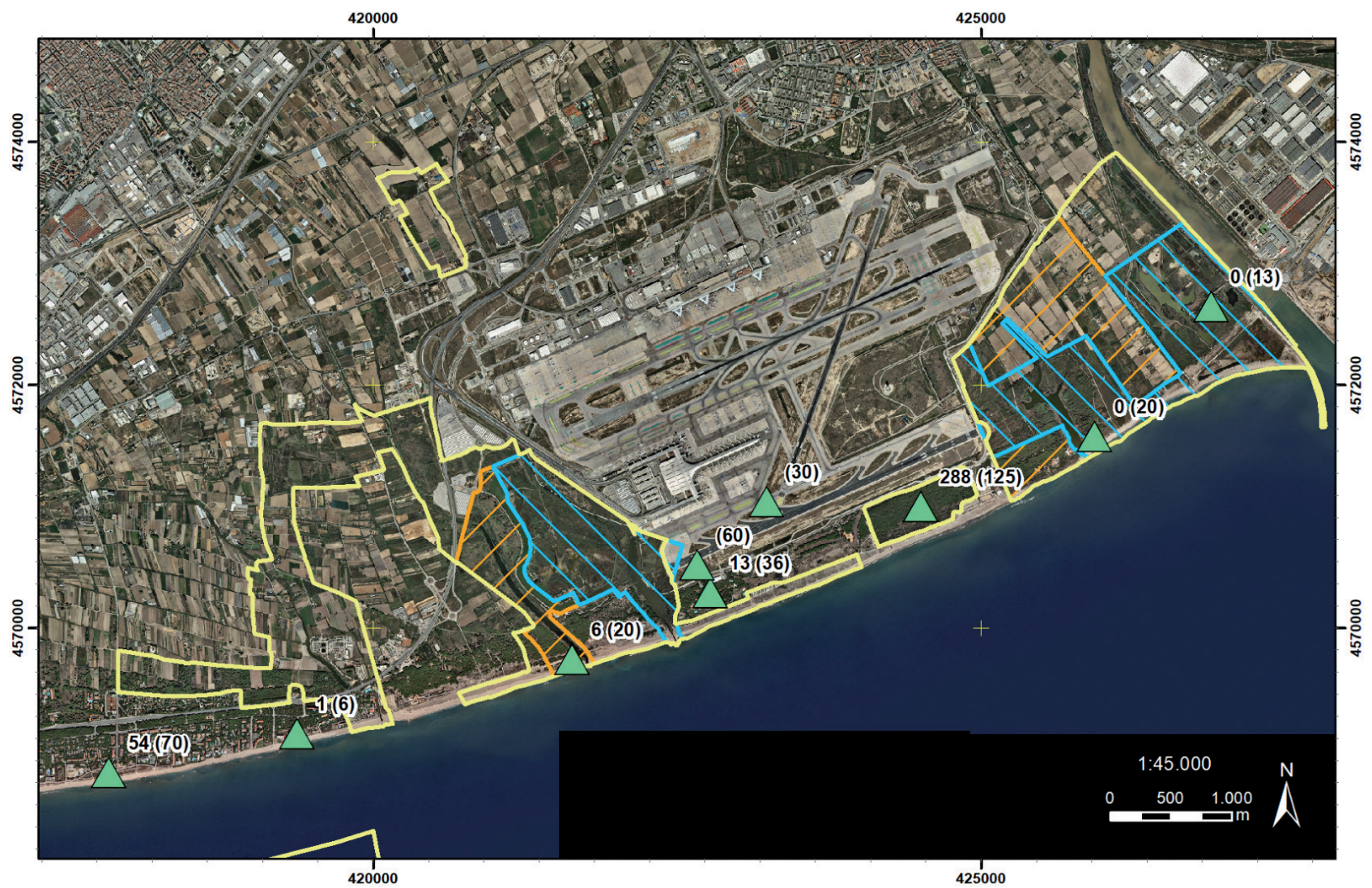

Figura 3. Mapa de las localidades prospectadas para Orobanche foetida (triangulo verde) en el hemidelta sur del río Llobregat. Los números indican el censo de individuos en 2018 y, entre paréntesis, el censo en 2003. También se indican los espacios protegidos: Red Natura 2000 (línea amarilla), Reserva natural parcial (trama azul) y Zona periférica de la reserva natural parcial (trama naranja)

Bibliografía

- Buord, S., D. Gargano \& J.C. Moreno Saiz (2011). Kosteletzkya pentacarpa. The IUCN Red List of Threatened Species 2011. e.T161916A5513198.

- González, V., R. del Hoyo, J.M. Seguí \& A. Valverde (2016). Flora vascular del Delta del Llobregat. Treballs de la Institució Catalana d'Història Natural.

- Pino, J. (2003). Distribució i cens d'Orobanche foetida Poir al delta del Llobregat. Butlletí de la Institució Catalana d'Història Natural 71: 129-134
- Pino, J., F.X. Picó \& E. de Roa (2007). Population dynamics of the rare plant Kosteletzkya pentacarpos (Malvaceae): a nine-year study. Botanical Journal of the Linnean Society 153: 455-462.

- Pino, J. \& E. de Roa (2003). Current census and distribution of Kosteletzkya pentacarpa (Malvaceae) in the Llobregat delta (Barcelona). Anales del Jardin Botánico de Madrid 60: 226-227.
- Pino, J. \& E. de Roa (2007). Population biology of Kosteletzkya pentacarpa (Malvaceae) in the Llobregat delta (Catalonia, NE of Spain). Plant Ecology 188: $1-16$.

- Sáez, LI. P. Aymerich \& C. Blanché (2010). Llibre vermell de les plantes vasculars endèmiques i amenaçades de Catalunya. Argania Ed., Barcelona, 811 p.

\section{Agradecimientos}

A E. de Roa, A. Ramal y X. Santaeufèmia, del Consorci per a la Protecció i la Gestió dels Espais Naturals del Delta del Llobregat, por su soporte técnico y a J.C. Moreno, J. Fuertes, M. Guardiola y M. Ramírez por su gran ayuda en las prospecciones.

LAURA FUENTES Y JOAN PINO

Centre de Recerca Ecològica i Aplicacions Forestals (CREAF) Universitat Autònoma de Barcelona. 08193 Bellaterra (Barcelona) 